\title{
On Tangent Bundles with Sasakian Metrics of Finslerian and Riemannian Manifolds.
}

\author{
Kentaro Yano (Japan) and (*) Tanjiro OkUbo (Canada) (**)
}

\begin{abstract}
On the basis of the so-called phase completion the notion of vertical, horizontal and complete objects is defined in the tangent bundles over Finslevian and Riemannian manifold. Such a tangent bundle is made into a manifold of almost Kaehlerian stru. cture by endowing it with Sasakian metric. The components of curvature tensors with respect to the adapted frame are presented. This having been done it is shown possible to study the differential geometry of Finslerian spaces by dealing with that of their own tangent bundles.
\end{abstract}

\section{Introduction.}

There exist several essentially different points of view with regard to Finslerian spaces. C. OARATHEDORY [2] dealt with the spaces by means of variational calculus, while since 1925 J. L. Synge [17] and L. Berward [1] developed tensor calculus by understanding that Finslerian space is a manifold with a metric tensor whose components are the second derivatives of $\frac{1}{2} F^{2}(x, d x)$ where $F$ is a function satisfying the properties that it is positive, homogeneous of degree one in the differentials and convex in the latter. Regarding the differentials involved in $F$ as the components of the element of support and providing with the socalled base connection, which we call $\delta_{i}$-derivation. E. CARTAN [3] endowed Finslerian spaces with Euclidean connection. The metric tensor being functions of position and element of support, a trend arose to observe that Finslerian space can be derived from a Riemannian space by the application of homogeneous contact transformation [6], [8] which by K. Y YNo and E.T. DAvies [19] was elevated to the contact tensor calculus. There the special frame of reference called the first and second contact frames introduced by M. S. KNEBEIMAN [9] and Y. Muto [13], respectively, served to define the sub-distributions complementary and non-holonomic in general.

Recently the present authors [24] developed the differential geometry of tangent bundles over affinely connected spaces of Finslerian type, which are called generalized spaces of paihs $[6],[11]$ and showed that it is always pos. sible to regard the geometry of such a space as that of its tangent bundle

$\left.{ }^{*}\right)$ This work was supported by National Research Coundil of Canada A-4037 (1960-70).

(**) Entrata in Redazione 1'8 marzo 1970. 
by means of the so-ealled phase completion. There the definition of horizontal distribution was given by the use of E. OARTAs's base connection whieh helped to define the first contact trame in the contact tensor calculus. Thus the geometry of spaces of Finslerian type lies in this category needless to say.

However, the problem we face consists in giving the metrics in tangent bundles. For though it would be very natural for which to use the so-called vertical, horizontal and complete tensor fields of type (0.2) that arise from the Finsler metrie $g$ derived from the fundamental function $F$ of the base manifold, either of them renders the tangent bundle unable to be a proper Riemannian manifold. On the other hand $\mathrm{S}$. SASAKI [16] introduced a special metric $g^{S}$ so that the tangent bundle over a Riemannian manifold may be a proper Riemannian manifold, and later on K. Yano and E. T. Davies adopted this scheme to study the differential geometry of the tangent bundles over Finslerian and Riemannain manifolds [21]. The purpose of the present paper

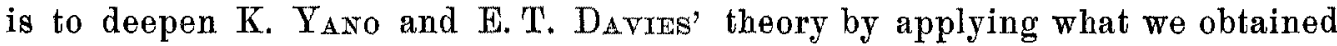
in our theory of tangent bundles over generalized spaces of paths. The Sasakian metric $g^{S}$ makes them into almost Kaehlerian manifolds and we prove that they can not be Einstein manifolds. Also we derive the curvature properties of base Finslerian manifolds uniformly by computing the curvature tensor of their tangent bundles, which may by all means be one of the contributions to the theory of tangent bundles to Finsler geometry.

\section{§ 1. - Tangent bnndle over a Finslerian manifold.}

Let $M$ be an $n$-dimensional manifold whose class of differentiability is assumed to be as high as required. Then its tangent bundle $T(M)$ is by definition

$$
T(M)=\bigcup_{P \in M} T_{P}(M)
$$

where $P$ is a point of $M$ and $T_{P}(M)$ is the tangent plane of $M$ at $P$. A point $\tilde{\boldsymbol{P}}$ of $T(\boldsymbol{M})$ is an ordered pair $\left(P, y_{P}\right)$ of a point $P$ and a vector $y_{P} \in T_{P}(M)$. $\pi$ is the projection $T(M) \rightarrow M$ defined by $\tilde{P}=\left(P, y_{P}\right) \rightarrow P$. The set $\pi^{-1}(P)$ is called the fibre over $P$, and $M$ is the base manifold.

Suppose that the manifold $M$ is covered by a system of coordinate neigh. bourhoods $\left\{U, x^{h}\right\}\left(^{1}\right)$, where $\left(x^{h}\right)$ is the local coordinate systems in the neighbourhood $U$. Let $\left(y^{h}\right)$ be the system of Cartesian coordinates in each tangent space $T_{p}(M)$ of $M$ with respect to the natural base $\left(\partial_{h}\right)$, where $\partial_{h}=\partial / \partial x^{h}$. Then in the open set $\pi^{-1}(U)$ of $T(M)$ we can introduce local coordinates $\left(x^{h}, y^{h}\right)$ for $\tilde{P}$, which we call coordinates in $\pi^{-1}(U)$ induced from $\left(x^{h}\right)$, or simply induced coordinates in $\pi^{-1}(U)$.

(1) The indices $a, b, c, d, \ldots, h, i, j, k, \ldots$ run over the range $\{1,2, \ldots, n\}$. 
If $U^{\prime}$ is another coordinate neighbourhood of $P$ in $M$, then $\pi^{-1}\left(U^{\prime}\right)$ contains $\tilde{P}$ and the induced coordinates of $\tilde{P}$ relative to $\left.\pi^{-1} U^{\prime}\right)$ are $\left(x^{h^{\prime}}, y^{h^{\prime}}\right)$, where

$$
x^{h^{\prime}}=x^{h^{\prime}}(x)
$$

On writing (1.2) shortly as

$$
y^{h^{\prime}}=\partial_{h} y^{h^{\prime}} \cdot y^{h}
$$

$$
x^{A^{\prime}}=x^{A^{\prime}}(x)\left({ }^{2}\right)
$$

where we understand that $x^{\bar{h}}=y^{h}$ and $x^{\overline{h^{\prime}}}=y^{h^{\prime}}$, the Jacobian of the transformation (1.3) is given by

$$
\left(\partial_{B} x^{A^{\prime}}\right)=\left[\begin{array}{lr}
\partial_{i} x^{h^{\prime}} & 0 \\
y^{a} \partial_{a} \partial_{i} x^{h^{\prime}} & \partial_{i} x^{h^{\prime}}
\end{array}\right] .
$$

Thus the tangent bundle $T(M)$ is orientable [21].

We suppose that there is given in $T(M)$ a function $F(x, y)$ satisfying the properties

(a) $F(x, y)>0$ for $y \neq 0$,

(b) $F(x, \alpha y)=\alpha F(x, y)$ for real $\alpha$,

(c) $F(x, y+z)=F(x, y)+F(x, z)$,

(d) For $x^{h}=x_{0}^{h}$, the surface $F\left(x_{0}, y\right)=1$ is a convex surface.

Then it is clear that the $n^{2}$ symmetric function $g_{j i}(x, y)$ defined in $\pi^{-1}(U)$ by

$$
g_{j i}(x, y)=\partial^{2} \frac{1}{2} F^{2} / \partial y^{j} \partial y^{i}
$$

undergo the law of transformation

$$
g_{j^{\prime} i^{\prime}}=\partial_{j^{\prime}} x^{j} \cdot \partial_{i^{\prime}} x^{i} \cdot g_{j i}
$$

subject to (1.3), and when $y^{h}$ is replaced by the direction $\dot{x}^{h}=\frac{d x^{h}}{d t}$ of a point $P\left(x^{h}\right) \in U, g_{j i}$ can make $M$ into a Finslerian space. Then $T(M)$ is the tangent bundle over a Finslerian manifold $M$.

(*) The indices $A, B, C, D, E, \ldots$ run over the range $\mid 1,2, \ldots, n ; \overline{1}, \overline{2}, \ldots, \bar{n}$, 
We suppose moreover that in each $\pi^{-1}(U)$ there are given $n^{2}$ functions $\Gamma_{i}^{h}(x, y)$ satisfying the properties

(a) $\Gamma_{i}^{h}(x, \alpha y)=\alpha \Gamma_{i}^{h}(x, y)$

(b) $\Gamma_{i}^{h}(x, y)$ undergo the law of transformation

$$
\Gamma_{i^{\prime}}^{h^{\prime}}\left(x^{\prime}, y^{\prime}\right)=\partial_{h} x^{h^{\prime}} \cdot\left(\partial_{i} x^{i^{\prime}} \cdot \Gamma_{i}^{h^{h}}+y^{a} \partial_{a^{\prime}} \partial_{j^{\prime}} x^{h^{\prime}}\right),
$$

subject to (1.3).

By the use of $\Gamma_{i}^{h}$ we introduce the operator $\delta_{i}$ defined by

$$
\delta_{i}=\partial_{i}-\Gamma_{i}^{a} \partial_{a}
$$

then for any function $f(x, y)$ of class $C^{r}, r \geq 1, \delta_{i} f$ can be defined in $T(M)$ globally in virtue of (1.2), (1.7) and

$$
\partial_{i} f=\partial_{i^{\prime}} x^{i} \cdot \partial_{i} f+\partial_{i} y^{a} \cdot \partial_{a} f
$$

Since each of $\delta_{i}$ and $\partial_{i}$ serves to be the base of $T(M)$, and denoting them respectively by $B_{i}^{A}$ and $C_{i}^{A}$, we introduce in $T(M)$ a special frame of reference $A_{\alpha}^{A}=\left(B_{i}^{A}, O_{i}^{A}\right)$, which we call adapted frame [21].

$$
B_{i}^{A}=\left(\delta_{i}^{h}-\Gamma_{i}^{h}\right), C_{i}^{A}=\left(0, \delta_{i}^{h}\right) .
$$

The dual frame $B_{B}^{\mathrm{P}}=\left(B_{B}^{h}, C_{B}^{\bar{h}}\right)$ has the components inverse to $A_{\alpha}^{A}$ and they are given by

$$
B_{B}^{h}=\left(\delta_{i}^{h}, 0\right), C_{B}^{\bar{h}}=\left(\Gamma_{i}^{h}, \delta_{i}^{h}\right)
$$

Obviously the equations of a fibre are given by

$$
d x^{h}=B_{B}^{h} d x^{B}=0
$$

while its complementary sub-distribution is given by $n$ equations

$$
C_{B}^{\bar{k}} d x^{B}=d y^{h}+\Gamma_{i}^{h}(x, y) d x^{i}=0
$$

and it is called the horizontal distribution of $T(M)$.

The non-holonomic object with respect to $A_{\alpha}^{A}$ is given by

$$
\left.\Omega_{\gamma \beta}^{\alpha}=-\Omega_{\beta \gamma}^{\alpha}=A_{A\left(\delta_{\alpha}\right.}^{\alpha} A_{\beta}^{A}-\delta_{\beta} A_{\gamma}^{A}\right)
$$


where

$$
\delta_{\alpha}=\delta_{i} \text { for } \alpha=i, \text { and } \delta_{\alpha}=\delta_{\bar{i}} \text { for } \alpha=\bar{i}
$$

and the non-vanishing components of $\Omega_{r \beta}^{\alpha}$ will be

$$
\begin{aligned}
& \Omega_{j i}^{h}=-\Omega_{i j}^{h}=-\left(\delta_{i} \Gamma_{i}^{h}-\delta_{i} \Gamma_{j}^{h}\right) \\
& \Omega_{j i}^{h}=-\Omega_{j i}^{h}=-\delta_{j} \Gamma_{i}^{h} .
\end{aligned}
$$

Let $C: x^{h}=x^{h}(t)$ be a curve of class $C^{r}, r \geq 1$, in $U$ of $M$ and suppose that its natural lift $\left(x^{h}, \dot{x}^{h}\right)$ in $\pi^{-1}(U)$ lies in horizontal distribution always, that is to say, $x^{h}(l)$ satisfies the equations

$$
\frac{d^{2} x^{h}}{d t^{2}}+\Gamma_{j i}^{h}(x, \dot{x}) \frac{d x^{j}}{d t} \frac{d x^{i}}{d t}=0
$$

where,

$$
\Gamma_{j i}^{h} \dot{x^{i}}=\Gamma_{j}^{h}
$$

then we call $C$ the generolized geodesic of $M$, or simply the geodesic.

In $M$ we take a vec or field $X$ along such geodesic $C$ and consider the infinitesimal transforma on

$$
\bar{x}^{h}(t)=x^{h}(t)+X^{h}(x(t)) \delta u,
$$

where $X^{h}(x(t))$ are the components of $X$ with respect to $\left(\partial_{h}\right)$. Then the direetion $\dot{x}(t)$ of $O$ is transformed into

$$
\dot{\overline{x^{h}}}(t)=\dot{x}^{h}(t)+X^{h}, \dot{x}^{j}(t) \delta u .
$$

We say that if the transformation (1.14) carries $C$ to a path of $M$ within the preservation of the parameter $t$, the vector field $X$ defines an affine collineation. The necessary and sufficient condition for $X$ to define an affine collineation is the vanishing of the lie derivatives $\mathfrak{S}_{X} \Gamma_{j i}^{h}[11],[14],[20]$ :

$$
\begin{aligned}
& \mathfrak{S}_{X} \Gamma_{j i}^{h}(x, \dot{x})=\partial_{j} \partial_{i} x^{h}-\Gamma_{j i}^{a} \partial_{a} \nabla^{h}+\Gamma_{j a}^{h} \partial_{i} \nabla^{a}+\nabla^{a} \partial_{a} \Gamma_{j i}^{h} \\
& +\Gamma_{j i}^{h} \dot{x}^{b} \partial_{b} \nabla^{a} \\
& =\nabla_{j} \nabla_{i} X^{h}+K_{k j i}^{h} X^{k}+\Gamma_{j i a}^{h} \dot{x}^{b} \nabla_{b} X^{a}=0,
\end{aligned}
$$

where we have put

$$
\nabla_{j} X^{h}=\delta X^{h}+\Gamma_{j a}^{h} X^{a}
$$




$$
K_{k j i}^{h}(x, \dot{x})=\delta_{k} \Gamma_{j i}^{h}-\delta_{j} \Gamma_{k a}^{h}+\Gamma_{k a}^{h} \Gamma_{j i}^{a}-\Gamma_{a j}^{h} \Gamma_{k i}^{a}
$$

and we notice that $\delta_{j} X^{h}(x(t))=\partial_{j} X^{h}(x(t))$ for the case.

Let $X^{h}(x, y)$ and $\omega_{i}(x, y)$ be two set of functions defined in $\pi^{-1}(U)$ such that they obey the law of transformation

$$
X^{h^{\prime}}=\partial_{h} x^{h^{\prime}} \cdot \omega_{i^{\prime}}=\partial_{i^{\prime}} x^{i} \cdot \omega_{i}
$$

subject to (1.31). Since such functions can always be defined in $U$ of $M$ by replacing the $y^{\prime} s$ by $x^{\prime} s$ as the standard work of defining vectors and 1-forms in Finsler geometry, our concern is to define any vector or 1-form in $T(M)$ by the use of these $n$ functions given a priori. Such an action may be called phase completion of the tangent bundle over a Finslerian manifold. There would be many ways for phase completion, but we shall deal with the following three kinds. We define the vertical, horizontal and complete vector fields denoted respectively by $X^{V}, X^{H}$ and $X^{C}$ which have in $\pi^{-1}(U)$ the com. ponents

$$
X^{V}=\left(\begin{array}{c}
0 \\
X^{h}
\end{array}\right), X^{H}=\left(\begin{array}{c}
X^{h} \\
-\Gamma_{i}^{h} X^{i}
\end{array}\right), X^{c}=\left(\begin{array}{c}
X^{h} \\
y^{a} \delta_{a} X^{h}
\end{array}\right)
$$

and the vertical, horizontal and complete 1-form's denoted respectively by $\omega^{V}$, $\omega^{H}$ and $\omega^{C}$ which have in $\pi^{-1}(U)$ the components

$$
\omega^{V}=\left(\omega_{i}, 0\right), \omega^{H}=\left(\omega_{i}, \Gamma_{i}^{h} \omega_{k}\right), \omega^{c}=\left(y^{a} \delta_{a} \omega_{i}, \omega_{i}\right)
$$

where each components are expressed in terms of the natural base $\left(\partial_{A}\right)$.

Let $P$ and $Q$ be the two sets composed respectively of $n^{r+s}$ functions

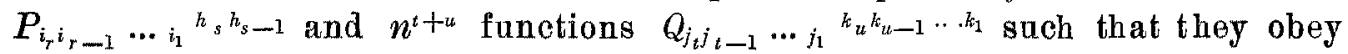
the law of transformation

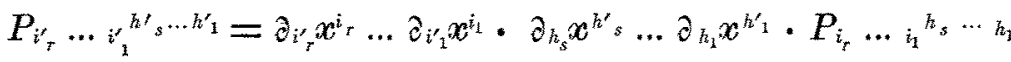

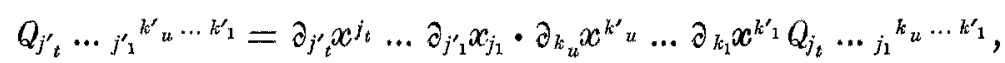

and denote by $P \times Q$ the formal product of these two sets of functions.

We define the vertical, horizontal and complete tensor fields by

respectively [24].

$$
\begin{aligned}
& (P \times Q)^{V}=P^{V} \otimes Q^{V}, \\
& (P \times Q)^{H}=P^{H} \otimes Q^{V}+P^{V} \otimes Q^{H}, \\
& (P \times Q)^{C}=P^{C} \otimes Q^{V}+P^{V} \otimes Q^{C},
\end{aligned}
$$


From these definitions the vertical, horizontal and complete tensor fields $G$ of type $(0.2)$ have in $\pi^{-1}(U)$ the components

$$
G^{V}=\left(\begin{array}{cc}
G_{j i} & 0 \\
0 & 0
\end{array}\right), G^{H}=\left(\begin{array}{cc}
\Gamma_{j}^{c} G_{t i}+\Gamma_{i}^{t} G_{j t} & G_{j i} \\
G_{j i} & 0
\end{array}\right), G^{C}=\left(\begin{array}{cc}
y^{a} \widehat{\delta}_{a} G_{j i} & G_{j i} \\
G_{j i} & 0
\end{array}\right)
$$

respectively. Thus, on taking $g_{j i}$ given by (1.5), we can metrize $T(M)$ by $g^{c}$, However, such $g^{c}$ makes $T(M)$ into a pseudo Riemannian manifold [23], and we now introdace in $T(M)$ a special metric $g^{S}$ which we call Sasakian lift of the metric $g$ of $M$ and was adapted by S. Sas.akr [16] in order to make the tangent bundle over a Riemannian manifold with the metric $g_{j i}(x)$ into property Riemannian. On putting $g_{j s} \Gamma_{j a}^{s} y^{a}=\Gamma_{i j}$, it is given by

$$
g^{s}=\left(\begin{array}{cc}
g_{j i}+g_{c b} \Gamma_{j}^{c} \Gamma_{i}^{b} & \Gamma_{i j} \\
\Gamma_{j i} & g_{j i}
\end{array}\right)
$$

and with respect to the adapted frame it has the components

$$
g^{S}=\left|\begin{array}{cc}
g_{j i} & 0^{-} \\
0 & g_{j i}
\end{array}\right|
$$

and consequently the element of are length in $T(M)$ is given by

$$
d S^{2}=g_{j i} d x^{j} d x^{i}+g_{i i} \delta y^{j} \delta y^{i}
$$

Thus we have proved.

THEOREM 1. - The tangent bundle $T(M)$ over a Finsler space $M$ is a Riemannian manifold with respect to Sasakian lift $g^{S}$.

Denoting by $G^{S}$ the inverse to $g^{S}$, we find $G^{S}$ has the components

$$
G^{s}=\left(\begin{array}{cc}
g^{i h} & -\Gamma^{h i} \\
-\Gamma^{i \hbar} & g^{i \hbar}+g^{c b} \Gamma_{c}^{h} \Gamma_{b}^{i}
\end{array}\right)
$$

with respect to the natural base $(\partial A)$ and

$$
G^{S}=\left[\begin{array}{cc}
g^{i h} & 0^{-} \\
0 & g_{\text {ih }}
\end{array} \mid\right.
$$

with respect to the adapted frame, where $g_{j i} g^{i h}=\delta_{i}^{h}$. 
$\S 2,-$ Euclidean connection in $T(M)$.

$T(M)$ having been made into a proper Riemannian manifold, there exists a unique connection $\nabla^{S}$ that keeps $g^{S}$ covariantly constant and is torsion free. It is given by [12]

$$
\begin{aligned}
& 2 g^{S}\left(\nabla^{\mathcal{X}} \tilde{Y}, \tilde{Z}\right)=\tilde{X} g^{s}(\tilde{Y}, \tilde{Z})+\tilde{Y} g^{S}(\tilde{Z}, \tilde{X})-\tilde{Z} g^{S}(\tilde{X}, \tilde{Y}) \\
& \quad+g^{s}([\tilde{X}, \tilde{Y}], \tilde{Z})+g^{S}([\tilde{Z}, \tilde{X}], \tilde{Y})+g^{s}(\tilde{X},[\tilde{Z}, \tilde{Y}])
\end{aligned}
$$

where $\tilde{X}, \tilde{Y}$ and $\tilde{Z}$ are any vector fields of $T(M)$, and in terms of the local coordinates $\left(x^{h}, y^{h}\right)$ of $\pi^{-1}(U)$ the parameter $\Gamma_{C B}^{A}$ of $\Gamma^{S}$ is the Cristoffel symbol. Its corresponding coefficients with respect to the adapted frame are given by

$$
\Gamma_{\gamma \beta}^{\alpha}=A_{A}^{\alpha}\left(\delta_{\gamma} A_{\beta}^{A}+\Gamma_{C B}^{A} A_{\gamma}^{C} A_{\beta}^{B}\right)
$$

whence

$$
\Gamma_{Y \beta}^{\alpha}-\Gamma_{\beta \gamma}^{\alpha}=\Omega_{Y \beta}^{\alpha}
$$

The covariant derivative of $g_{\gamma \beta}^{S}$ is given by

$$
\nabla \delta g_{\uparrow \beta}=\delta_{\delta} g_{\gamma \beta}-\Gamma_{\delta \gamma}^{\varepsilon} g_{\varepsilon \beta}-\Gamma_{\delta \beta}^{\varepsilon} g_{\curlyvee \varepsilon}=0
$$

from which we obtain

$$
\Gamma_{\gamma \beta}^{\alpha}=\frac{1}{2} g^{\alpha \varepsilon}\left(\delta_{\gamma} g_{\varepsilon \beta}+\delta_{\beta} g_{\gamma^{\beta}}-\delta_{\varepsilon} g_{\gamma \beta}\right)+\frac{1}{2}\left(\Omega_{\tau \beta}^{\alpha}+\Omega_{\tau \beta}^{\alpha}+\Omega_{\cdot \beta_{\gamma}}^{\alpha}\right)
$$

where we have written

$$
\Omega^{\alpha} \cdot \Upsilon \beta=g^{\imath \delta} g_{\delta \beta} \Omega_{\mathrm{\varepsilon} \gamma}^{\delta} \text {. }
$$

The particular values of $\Gamma$ for different indices, on taking account of (1.13) and (1.24), are found to be
(a) $2 \Gamma_{j i}^{h}=g^{h a}\left(\delta_{j} g_{i a}+\delta_{i} g_{j a}-\delta_{n} g_{j i}\right)$
(b) $2 \Gamma_{\bar{j} i}^{h}=g^{h a \partial_{j}} g_{i a}+\Omega^{h}{ }_{i \bar{j}}$,
(c) $2 \Gamma_{j \bar{i}}^{h}=g^{h a} \partial_{i} g_{j a}+\Omega^{h}{ }_{j \bar{i}}$
(d) $2 \Gamma_{\tilde{j}_{\bar{i}}}^{h}=-g^{h a} \delta_{a} g_{j i}+\Omega^{h}{ }_{\bar{j} \bar{i}}+\Omega^{h}{ }_{\bar{i} \bar{j}}$. 
$(2.7)$
(e) $2 \Gamma_{j i}^{\bar{h}}=-g^{h a} \partial_{a} g_{j i}+\Omega_{j i}{ }^{\bar{h}}$
(f) $2 \Gamma_{\bar{j}}^{\bar{h}}=g^{h a} \hat{\delta}_{i} g_{j a}+\Omega_{\bar{j}}{ }^{\bar{h}}+\Omega^{\bar{h}}{ }_{i \bar{j}}$,
(g) $2 \Gamma_{j \bar{i}}^{\bar{h}}=g^{h a \delta_{j}} g_{i a}+\Omega_{j \bar{i}} \bar{h}+\Omega_{j \bar{i}}^{\bar{h}}$,
(h) $2 \Gamma_{\bar{j} \bar{i}}^{\bar{h}}=g^{h a} \partial_{j} g_{i a}$.

If we use the notations

$$
\begin{aligned}
& 2\left\{\begin{array}{c}
h \\
j i
\end{array}\right)=g^{h a}\left(\partial_{j} g_{a i}+\partial_{i} g_{j a}-\partial_{a} g_{j i}\right), \\
& 2 C_{j i h}=\partial_{j} g_{j h},
\end{aligned}
$$

the $(2.7 \mathrm{a})$ becomes

$$
\Gamma_{j i}^{h}=\left\{\begin{array}{c}
h \\
j i
\end{array}\right\}-g^{h a}\left(\Gamma_{j}^{b} C_{b i a}+\Gamma_{i}^{b} C_{b j a}-\Gamma_{a}^{b} C_{b j i}\right)
$$

Taking account of homogeneity properties of $C^{\prime} s$, we have immediately

$$
\Gamma_{j i}^{h} y^{i} y^{i}=\left\{\begin{array}{c}
h \\
j i
\end{array}\right\} y^{j} y^{i} \equiv 2 G^{h}
$$

and if we now take

$$
\Gamma_{i}^{h}=3_{\bar{i}} G^{h},
$$

we have an expression on the right hand side of $(2.10)$ as

$$
\Gamma_{j i}^{h}=\left\{\begin{array}{c}
h \\
j i
\end{array}\right\}-\left(\partial_{j} G^{a}\right) C_{i a}{ }^{h}-\left(\partial_{\bar{i}} G^{a}\right) C_{j a}{ }^{h}+g^{h a}\left(\partial_{a}^{-} G^{b}\right) C_{b j i},
$$

which coincides with the expression for $\Gamma^{*}$ in Cartian's theory [3].

The fact that

$$
\begin{gathered}
\Gamma_{i}^{h}=\Gamma_{j i}^{h} y^{j}, \\
\delta_{k} F=\partial_{k} F^{h}-\Gamma_{b i}^{a} \partial_{a}^{-} F=0, \\
y^{a} \partial_{j} \Gamma_{a i}^{h}=y^{a} \partial_{\bar{i}} \Gamma_{j a}^{h}=y^{a} \nabla_{a} C_{j i}^{h}
\end{gathered}
$$

are easily verified. 
With (2.16)' we can determine the non-vanishing components of the nonholonomic objects that were given in (1.14). For $\Omega_{j i}^{h}$ we have

$$
\Omega_{j i}{ }^{h}=-K_{j i a}{ }^{h} y^{a}
$$

where $K_{k j i}^{h}$ are those given in (1.21) whose variables $\dot{x}^{\prime} s$ are replaced by the $y^{\prime} s$.

Similarly

$$
\Omega_{j b^{k}}^{h}=-\partial_{j} \partial_{i}^{-} G^{h} \equiv G_{j i}{ }^{h}
$$

From this definition we have

$$
g_{h a} G_{j i}^{a}=g_{h a} \Gamma_{j i}^{a}+\left(\hat{\varrho}_{j} g_{h a} \Gamma_{i b}^{a}\right) y^{b}-2 C_{j k a} \Gamma_{i b}^{a} y^{b}
$$

from which, substituting from (2.10), we have

or

$$
g_{h a} G_{j i}^{a}=g_{h a} \Gamma_{j i}^{a}+y^{a} \nabla_{a} C_{j i \hbar}
$$

$$
\Omega_{j i}{ }^{h}=G_{j i}{ }^{h}=\Gamma_{j i}^{h}+\nabla_{0} A_{j i}{ }^{h},
$$

where

$$
A_{j i^{h}}=F C_{j i}{ }^{h}, \quad \nabla 0=\frac{1}{F} y^{a} \nabla_{a} .
$$

We can now rewrite (2.7) in the form $\left({ }^{3}\right)$
(a) $\Gamma_{j i}^{h}=\Gamma_{j i}^{* h}$ of Cartan,
(b) $2 \Gamma_{j i}^{h}=2 C_{j i}^{h}-K^{h}{ }_{i a j} y^{a}$,
(c) $2 \Gamma_{j i}^{h}=2 C_{j i}{ }^{h}-K_{j a i}^{h} y^{a}$,
(d) $\Gamma_{\bar{j}_{i}}^{h}=\nabla_{0} A_{j i}{ }^{h}$,
(e) $2 \Gamma_{j i \bar{t}}^{\bar{h}}=-2 C_{j i}^{h}-K_{j i a}^{h} y^{a}$,
(f) $\Gamma_{\bar{j}, \bar{h}}^{\bar{h}}=-\nabla_{0} A_{j i}{ }^{h}$,
(g) $\Gamma_{j \bar{i}}^{\bar{h}}=\Gamma_{j k}^{h}$,
(h) $\Gamma_{\bar{j} \bar{i}}^{\bar{k}}=C_{j i}{ }^{n}$,

(3) In the formulas of $\Gamma^{\prime} s$ enlisted as (3.21) by K. Yano and E. T. Davies' paper [21], the plus sign printed in $(b)$ and $(c)$ should be read to be the minus sign as seen above cor. respondingly. 
If we construct the vector field $A_{i}$ by contraoting $A_{j i}{ }^{h}$ given in (2.18) with respect to $j$ and $h$, we have

$$
A_{i}=A_{j i}{ }^{j}=\frac{1}{2} F \partial_{i} \log g, g=\operatorname{det} .\left(g_{j i}\right),
$$

and with regard to which A. DEIKE [5] proved.

Lemma. - If in a Finslerian space the $n$ functions $A_{i}$ which are equal to $\frac{1}{2} F \mathfrak{a}_{i} \log g$ vanish identically, then the space is Riemannian.

For the case, the function $F(x, y)$ defined in $\$ 1$ becomes

and $\Gamma^{\prime} s$ in (2.19) reduce to

$$
F^{2}=g_{j i}(x) y^{j} y^{i}
$$
(a) $\quad \mathbf{I}_{j i}^{h}=\left\{\begin{array}{c}h \\ j i\end{array}\right\}$
(b) $2 \Gamma_{j^{i} i}^{h}=-K_{j a i}{ }^{h} y^{a}$,
(c) $2 \Gamma_{j \dddot{i}}^{h}=-K_{i a j}^{h} y^{a}$,
(d) $\mathrm{T}_{\overline{j i}}^{h}=0$,
(e) $2 \Gamma_{j i}^{\bar{h}}=-K_{j i a}^{k} y^{a}$,
(f) $\Gamma_{\frac{\bar{j} i}{\bar{h}}}^{\bar{h}}=0$,
(g) $\Gamma_{j \bar{i}}^{\bar{b}}=\Gamma_{j i}^{h}$,
(h) $\quad \Gamma_{\bar{j} \bar{i}}^{\bar{h}}=0$.

If we express $X^{V}, X^{H}$ and $X^{C}$ given by (1.22) in terms of the adapted frame, they have the components

$$
X^{V}=\left[\begin{array}{c}
0 \\
X^{h}
\end{array}\right], X^{H}=\left[\begin{array}{c}
X^{h} \\
0
\end{array}\right], X^{C}=\left[\begin{array}{cc}
X^{h} \\
y^{a} \nabla & { }_{a} X^{h}
\end{array}\right] \text {. }
$$

respectively. When $M$ is Riemannian, the $X^{\prime} s$ appearing in (2.2) are the components of a vector field in $U$, and $X^{V}, X^{H}$ and $X^{C}$ are called the vertical, horizontal and complete lift of $X$ [16], [23]. 
If we compute the covariant derivatives of those vector fields, we have by using (2.19) for the vertical vector $X^{r}$

(a) $\nabla_{i}^{S} \tilde{X}^{\alpha}=\left[\left(C_{j i}^{k}-\frac{1}{2} K_{j b i}^{h} y^{b}\right) X^{i}, \nabla_{j} X^{h}\right]$,

(b) $\nabla_{j}^{S} \tilde{X}^{\alpha}=\left[\left(\nabla_{0} A_{j i}{ }^{h}\right) X^{i}, \partial_{j} X^{h}+C_{j i}{ }^{h} X^{i}\right]$,

and for the horizontal vector $X^{H}$

(a) $\nabla_{j}^{S} \tilde{X}^{\alpha}=\left[\nabla_{j} X^{h},-K_{j i a}^{h} y^{a} x^{i}\right]$,

(b) $\nabla_{\bar{j}}^{S} \tilde{X}^{\alpha}=\left[\left(C_{j i}^{h}-\frac{1}{2} K_{j a i}^{h} y^{a}\right) X^{i},-\left(\nabla_{0} A_{j i}^{\varpi^{h}}\right) X^{i}\right]$,

and. for the complete vector $X^{c}$

(a) $\nabla_{j}^{S} \tilde{X}^{a}=\left[\nabla_{j} X^{h}+\left(C_{j i}{ }^{h}-\frac{1}{2} K_{j a i}^{h} y^{a}\right) y^{b} \nabla_{b} X^{i}, \frac{1}{2} y^{a} \nabla_{j a} V^{h}+\right.$

$$
\left.+\frac{1}{2}\left(\nabla j \nabla a X^{h}+K_{b j a}{ }^{h} X^{b}\right)-C_{j i}{ }^{h} X^{i}\right] .
$$

(b) $\nabla_{j}^{S} \tilde{X}^{a}=\left[\partial_{j} X^{h}+\left(C_{j i}^{h}-\frac{1}{2} K^{h}{ }_{i a j} y^{a}\right) X^{i}-C_{j i}{ }^{h} y^{b} \nabla b{ }^{i}\right.$,

$$
\left.\partial_{j}\left(y^{a} \nabla_{a} X^{h}\right)+C_{j i}^{h}\left(y^{a} \nabla_{a} X^{i}\right)-X^{a} \nabla_{0} A_{j a}{ }^{h}\right] .
$$

Especially if $M$ is Riemannian we have for the vertical lift $X^{v}$

$$
\text { (a) } \nabla_{j}^{s} \tilde{X}^{\alpha}=\left[\frac{1}{2} y_{a \nabla a \nabla j} X^{h}-\frac{1}{2} y^{b \varrho_{X}}\left(\begin{array}{c}
h \\
j i
\end{array}\right), r_{j} X^{h}\right]
$$

(b) $\nabla \frac{s}{j} \tilde{X}^{\alpha}=[0,0]$,

and for the horizontal lift $X^{K}$

(a) $\nabla_{j}^{S} \tilde{X}^{\alpha}=\left[\nabla_{j}^{c} X^{h}, y^{\alpha} \varrho_{X}\left\{\begin{array}{c}h \\ a j\end{array}\right\}-y^{\alpha} \nabla j \nabla a X^{h}\right]$

(b) $\quad \nabla \frac{s}{j} \tilde{X}^{\alpha}=\left[\frac{1}{2} y^{a}(\nabla a \nabla j-\nabla j \nabla a) X^{h}, 0\right]$, 
and for the complete lift $X^{c}$.

(a) $\nabla_{j}^{S} \tilde{X}^{\alpha}=\left[\nabla_{j} X^{h}-\frac{1}{2} K_{i a j}^{h} y^{a} y^{b} \nabla_{b} X^{i}\right.$,

$$
\left.\frac{1}{2} y^{a} \nabla j \nabla a X^{h}+\frac{1}{2} y^{a} \hat{S}_{x}\left(\begin{array}{c}
h \\
j a
\end{array}\right)\right] \text {, }
$$

(b) $\quad \nabla \frac{s}{j} \tilde{X}^{\alpha}=\left[-\frac{1}{2} y^{a}(\nabla j \nabla a-\nabla a \nabla j) X^{h}, \nabla j X^{h}\right]$,

from which we obtain.

Theовем 2. - The verlical, horizontal and complete lifts of a vector field $X$ are parallel in $T(M)$ if and only if $X$ is a parallel vector field and defines an affine collineation.

\section{§ 3. - Almost complex structure of $T(M)$.}

Let $T(M)$ be the tungent bundle over a Finslerian manifold and have Sasakian lift $g^{S}$ as its metric.

We consider in $T(M)$ a tensor field $\tilde{F}$ of type (1.1) such that it acts on a vertical and horizontal vectors under the following rule:

$$
\tilde{F} X^{V}=X^{H} \quad \text { and } \quad \tilde{F} X^{H}=-X^{V} \text {. }
$$

Then we find that $\tilde{F}$ satisfies

$$
\tilde{F}^{2}=-\tilde{E}
$$

where $\tilde{E}$ is the unit tensor field in $T(M)$. Taking account of (2.2) they are found to have the components

$$
\tilde{F}^{1}=\left[\begin{array}{rr}
0 & \delta_{i}^{h} \\
-\delta_{i}^{h} & 0
\end{array}\right],
$$

and

$$
\tilde{E}=\left[\begin{array}{cc}
\delta_{i}^{h} & 0 \\
0 & \delta_{i}^{h}
\end{array}\right],
$$

with respect to the adapted frame. 
(3.2) or (3.3) implies that $\tilde{F}$ is an alnost complex structure [22] and has the components,

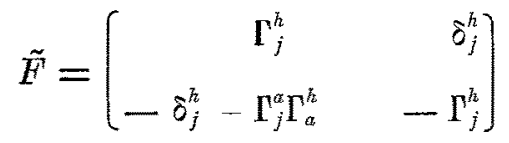

with respect to the natural frame $\left(\partial_{A}\right)$.

An using (1.23) and (1.26) if we compute the eomponents $F_{B A}$ of the tensor field $\tilde{F} g^{S}$ of type (1.1), they are found to be

$$
F_{B A}=\left(\begin{array}{cc}
\Gamma_{j i}-\Gamma_{i j} & g_{j i} \\
-g_{j i} & 0
\end{array}\right),
$$

where we have put

$$
\Gamma_{j i}=g_{i i} \Gamma_{b j}^{t} y^{b}
$$

and taking account of (2.10), (3.6) can be writen as

$$
F_{B A}=\left(\begin{array}{cc}
y^{\alpha}\left(\partial_{j} g_{i \alpha}-\partial_{i} g_{j a}\right) & g_{j i} \\
-g_{j i} & 0
\end{array}\right) .
$$

This implies that the exterior differential of the 1-form $\theta=g_{j i} y^{i} d x^{i}$ globally defined in $T(M)$ is

$$
d \theta=\frac{1}{2} F_{C B} d x^{C} \wedge d x^{B}
$$

and consequently $F_{A B}$ is closed. Thus we have

Theorem 3. - The tangent bundle $T(M)$ over a Finslerian or a Rieman. nian manifold $M$ whose metric is Sasakian lift $g^{S}$ is an almost Kaehlerian manifold.

The Nijenhais tensor $\tilde{N}$ of the almost complex structure $\tilde{F}$ is, by definition, given by [12]

$$
\tilde{N}(\tilde{X} \tilde{Y})=[\tilde{F} \tilde{X}, \tilde{F} \tilde{Y}]-\tilde{F}[\tilde{F} \tilde{X}, \tilde{Y}]-\tilde{F}[\tilde{X}, \tilde{F} \tilde{Y}]-[\tilde{X}, \tilde{Y}]
$$

where $\tilde{X}$ and $\tilde{Y}$ are arbitrary vector fields in $T(M)$, and $\tilde{N}$ has in $\pi^{-1}(U)$ the components of the form

$$
N_{C B}{ }^{A}=F_{C}^{D}\left(\partial_{D} F_{B}{ }^{A}-\hat{\partial}_{B} \bar{F}_{D}{ }^{A}\right)-F_{B}{ }^{D}\left(\partial_{D} F_{C}{ }^{A}-\partial_{C} F_{D}{ }^{A}\right)
$$

with respect to the natural frame. In terms of the adapted frame they are [21].

$$
\begin{aligned}
{[N]_{Y \beta^{\alpha}} } & =F_{Y}^{\delta}\left(\delta_{\delta} F_{\beta}^{\alpha}-\delta_{\beta} F_{\delta}^{\alpha}\right)-F_{\beta}^{\delta}\left(\delta_{\delta} F_{\gamma}^{\alpha}-\delta_{Y} F_{\delta}^{\alpha}\right) \\
& +F_{\varepsilon}^{\alpha}\left(F_{Y}^{\delta} \Omega_{\delta \beta^{\varepsilon}}-F_{\beta}^{\delta} \Omega_{\delta} \delta_{Y}^{\varepsilon}\right)+\left(\delta_{Y}^{\delta} \delta_{\beta}^{\delta}+F_{Y}{ }^{\varepsilon} F_{\beta}^{\delta}\right) \Omega_{\varepsilon \delta}^{\alpha} .
\end{aligned}
$$


On taking account of $(2.16),(2.17)$ and (3.3) if we compute the particular values of $N_{\gamma^{3}}$ for the different indices, we find that

$$
[N]_{j i}^{h}=[N]_{j i}^{h}=-K_{j i a}^{h} y^{a}
$$

all others being zero. Hence we have [18], [21].

Theorem 4. - The tangent bundle $T(M)$ over a Riemannian manifold $M$ having Sasakian lift $g^{S}$ as metric is a Kaehlerian manifold if and only if

$$
K_{j i a}^{h} y^{a}=0 .
$$

Tнеонем 5. - The tangent bundle $T(M)$ over a Riemannian manifold $M$ having Sasakian lift $g^{s}$ as metric is a Kaehlerian manifold if and only if $M$ is flat [21].

A vector $\tilde{X}$ with respect to which the tensor $\tilde{F^{i}}$ has the vanishing Lie derivative is said to be almost analytic. Denoting ay $\left[\mathcal{L}_{X} F\right]_{p}^{a}$ the components of $\mathfrak{S}_{X} F^{A}{ }_{B}$ with respect to the adapted frame, we have [21]

$$
\left[\mathcal{L}_{X} F^{\top}\right]_{\beta}^{\alpha}=X^{\varepsilon} \delta_{\varepsilon} F_{\beta}^{\alpha}-F_{\beta}^{\varepsilon} \delta_{\varepsilon} X^{\alpha}+F_{\varepsilon}^{\alpha} \delta_{\beta} X^{\varepsilon}+X^{\varepsilon}\left(\Omega_{\varepsilon \delta}^{\alpha} F_{\beta}^{\delta}-\Omega_{\varepsilon \beta}^{\delta} F_{\delta}^{\alpha}\right) .
$$

Then we obtain for the vertical vector $X^{V}$

$$
\begin{aligned}
& {\left[\mathfrak{2}_{X^{V}} W\right]_{j}{ }^{h}=\nabla_{j} X^{h}+X^{a} \nabla_{0} A_{a j}{ }^{h},\left[\mathfrak{S}_{X^{V}} F\right]_{j}^{h}=\hat{\jmath}_{j} X^{h},} \\
& {\left[\mathcal{L}_{X^{V}} X\right]_{j}^{\bar{h}}=\partial_{j} \boldsymbol{X}^{h},\left[\mathfrak{L}_{X^{V}} \boldsymbol{F}\right]_{j}^{\bar{j}}=-\nabla_{j} X^{h}-X^{a} \nabla_{0} A_{a j}{ }^{h} .}
\end{aligned}
$$

and for the horizontal vector $X^{H}$

$$
\begin{gathered}
{\left[\mathcal{L}_{X^{H}} \bar{H}\right]_{j}^{h}=\partial_{j} X^{h},\left[\mathcal{L}_{X^{H}} F\right]_{j}^{h}=-\nabla_{j} X^{h}-X^{a} \nabla^{0} A_{a j}{ }^{h},} \\
{\left[\mathcal{L}_{X^{H}} F\right]_{j}^{\bar{h}}=-\nabla_{j} X^{h}-X^{a} \nabla^{0} A_{a j^{h}}{ }^{h},\left[\mathcal{L}_{X^{H}} F\right]_{j}^{\bar{h}}=-\partial_{j}^{-} X^{h},}
\end{gathered}
$$

from which we have

Theorem 6. - The necessary and sufficient condition for the vertical vector $X^{r}$ or the horizontal vector field $X^{H}$ to be almost analytic is that $X^{h}$ satisfies in the base Finslerian manifold that

$$
\partial_{j} X^{h}=0, \quad \nabla_{j} X^{h}+X^{a} \nabla_{0} A_{a j}^{h}=0 .
$$

Theorex 7. - The necessary and sufficient condition for the vertical or horizontal lift of a vector fleld $X$ in the base Riemannian manifold to be almost analytic is that $X$ is a parallel vector field. 
As to the complete vector field $X^{c}$ we have

$$
\begin{aligned}
& {\left[\mathcal{L}_{X} c F\right]_{j}^{h}=\partial_{j} X^{h}+y^{a}\left\{\nabla j \nabla a X^{h}+K_{k j a}{ }^{h} X^{k}+\left(\partial-\Gamma_{a j}^{h}\right) y^{b} \nabla_{b} X^{c}\right\} \text {, }} \\
& {\left[\mathcal{L}_{X} c F\right]_{j}^{-h}=\left[\mathcal{L}_{X} c F\right]_{j}^{\vec{h}}=y^{a} \nabla_{a} \partial \vec{j} X^{h},} \\
& {\left[\varrho_{X} c F\right]_{j}^{\bar{h}}=y^{a}\left\{\nabla j \nabla a X^{h}+K_{k j a}{ }^{h} X^{k}+\left(\partial_{C} \Gamma_{a j}^{h}\right) y_{b}^{b} \nabla_{b} X^{c}\right\}}
\end{aligned}
$$

Therefore if $X^{h}$ are the components of a vector field $X$ defined along a geodesic $C$ of the base Finslerian manifold, then by taking account of (1.19), we have

Theonm 8. - The necessary and sufficient condition for the complete vector field $X^{C}$ lifted from a vector field $X$ defined along a geodesic $C$ in the base Finslerian manifold to be almost analytic is that $X$ defines an affine collineation.

We note that the equations of a geodesic given in (1.15) is, by taking the are tengths $S$ as parameter, written as [11], [15]

$$
\frac{d^{2} x^{h}}{d S^{2}}+\left\{\begin{array}{c}
h \\
j i
\end{array}\right\} \frac{d x^{i}}{d S} \frac{d x^{i}}{d S}=0
$$

because of $(2.10)$, and also that the statement similar to theorem 6 holds for the Riemannian case [21].

Let us consider the Lie derivative of the tensor $F_{C B}$. Denoting by $\left[\mathcal{L}_{X} F^{\prime}\right]_{C B}$ the components of ${ }_{X} F$ with respect to the adapted frame, we have

$$
\left[\mathcal{L}_{X} F\right]_{\gamma \beta}=X^{\delta \delta_{\delta}} F_{\gamma \beta}+F_{\delta \beta} \delta_{\gamma} X^{\delta}+F_{\gamma \delta} \delta_{\beta} X^{\delta}+X^{\varepsilon}\left(\Omega_{\varepsilon \gamma} \delta F_{\delta \beta}+\Omega_{\varepsilon \beta}{ }^{\delta} F_{\gamma \delta}\right),
$$

so that we have for the vertical vector field $X^{r}$

$$
\left[\mathcal{S}_{X^{V}} F\right]_{j i}=\nabla_{j} X_{i}-\nabla_{i} X_{j},\left[\varrho_{X} V F\right]_{j i}=-\nabla_{j} X_{i}
$$

where we have put

$$
\left[\mathcal{E}_{X} V F\right]_{\bar{j}}=0
$$

$$
X_{i}=g_{k i} X^{h}, \quad \nabla-X^{i}=\partial_{j} X_{i}-C_{j i}^{a} X^{a},
$$

and for the horizontal vector field $X^{H}$

$$
\left[\mathcal{E}_{X} H F\right]_{j i}=0, \quad\left[\mathcal{L}_{X^{H}} H F_{j i}\right]=\nabla_{j} X_{i}-X^{a} \nabla_{0} A_{j a i}
$$

$$
\left[\mathfrak{Q}_{X} H F\right]_{j i}=\partial_{i} X_{j}-\partial_{j} X_{i}
$$

from which we have for $T(M)$ over a Finslerian manifold $M$ 
Theonem 9, - The necessary and sufficient condition for (a) $\mathfrak{L}_{X} \boldsymbol{F}_{C B}$

(b) $\mathscr{S}_{X} H F_{C B}$ to vanish is that a) $\nabla_{j} X_{i}-\nabla_{i} X_{j}=0$ and

$$
\nabla_{j} X_{i}=0 \quad \text { (b) } \quad \nabla_{j} X_{i}=X^{a} \nabla{ }_{0} A_{j a i} \text { and } \partial_{j} X_{i}=\partial_{i} X_{j}
$$

and for $T(M)$ over a Riemannian manifold $M[21]$.

Theовем 10. - The necessary and sufficient condition for (a) $\mathfrak{L}_{X} \forall F_{C B}$

(b) $\mathfrak{S}_{X}{ }^{H} F_{C B}$ to vanish is that a) the vector field $X$ in $M$ is closed

(b) $X$ in $M$ is parallel.

As to the complete vector field $X^{c}$

$$
\begin{aligned}
& {\left[\mathcal{L}_{X} c F\right]_{j i}=g_{b j}\left(\nabla i \nabla a{ }^{b} X^{b}+K_{c i a}^{b} X^{c}+\left(\partial_{\bar{d}} \Gamma_{i a}^{b}\right) y^{c} \nabla^{d}\right\} y^{a}} \\
& \left.-g_{b i} \mid \nabla i \nabla_{a} X^{b}+K_{c j a}^{b} X^{c}+\left(\partial_{\bar{d}} \boldsymbol{\Gamma}_{j a}^{b}\right) y^{c} \nabla_{c} X^{d}\right\} \boldsymbol{y}^{a}, \\
& {\left[\mathfrak{S}_{X} c F\right]_{\bar{j} i}=\nabla_{j} X_{i}+\nabla_{i} X_{j}+C_{j i}{ }^{k} y^{a} \nabla_{a} X_{h_{b}},} \\
& {\left[\Omega_{X} C F\right]_{j} \bar{i}=\partial \bar{i} X_{j}-\partial_{j} X_{i} \text {. }}
\end{aligned}
$$

Let $X$ be a vector field defined along a curve $D: x^{h}=x^{h}(S)$ of class $C_{r}, r \geq 1$, and consider the infinitesimal transformation of the form given by (1.17). The necessary and sufficient condition for the transformation to preserve the are lengths of the curve $D$ is given by the vanishing of the Lie derivative $\mathfrak{S}_{X \mathbf{X}} g_{j^{3}}[11],[20]$ :

$$
\mathcal{L}_{X} g_{j i}(x, \dot{x})=\nabla_{i} X_{i}+\nabla_{i} X_{j}+C_{j i}{ }^{h} y^{a} \nabla_{a} X_{b}=0 .
$$

The vector field $X(x(t))$ of a Finslerian manifold which satisfies this condition is called Killing vector. If furthermore the Killing vector defines an affine collineation, we say that $X(x(S))$ generates motion [M.S. KNEBELMAN [11], p. 561].

Hence if in the above formula (3.17) the vector field $X^{C}$ is supposed to be constructed with the components of a vector field $X$ defined along a geodesic $C$ of $M$, we have

ThEonex 11. - The Lie derivative of $F_{C B}$ with respect to $X^{C}$ vanishes if $X$ generates a motion in the base Finslerian manifold. The similar statement holds for the Riemannian case too [21].

\$ 4. - Curvature tensor of $\nabla^{5}$.

Let $\tilde{X}, \tilde{Y}$ and $\tilde{Z}$ be any vector fields in $T(M)$. 
The curvature tensor $K^{S}$ of the connection $\Gamma^{s}$ is, by definition, given by

$$
K^{s}(\tilde{X}, \tilde{Y}) \tilde{Z}=[\nabla \stackrel{S}{\tilde{X}}, \nabla \stackrel{s}{\tilde{Y}}] \tilde{Z}-\nabla_{[\tilde{X}, \tilde{Y}]}^{s} \tilde{Z}
$$

and has in $\pi^{-1}(U)$ the components

$$
\left(K^{S}\right)_{D C B}{ }^{A}=\partial_{D} \mathbf{\Gamma}_{C B}{ }^{A}-\partial_{C} \boldsymbol{\Gamma}_{D B}{ }^{A}+\Gamma_{D E}^{A} \Gamma_{C B}^{E}--\boldsymbol{\Gamma}_{C E}{ }^{A} \Gamma_{D B}^{E} .
$$

If we express the Equation (4.1) in terms of the adapted frame, we have

$$
(\nabla \delta \nabla \gamma-\nabla \gamma \nabla \delta) Z^{x}=\left[K^{s}\right]_{\delta \curlyvee \beta}{ }^{x} Z^{\beta}
$$

where

$$
\left[K^{S}\right]_{\delta \gamma \beta}{ }^{\alpha}=\delta_{\delta} \Gamma_{\gamma \beta}^{\alpha}-\delta_{\gamma} \Gamma_{\delta \beta}^{\alpha}+\Gamma_{\delta \varepsilon}^{\alpha} \Gamma_{\gamma \beta}^{\varepsilon}--\Gamma_{\gamma \varepsilon}^{\alpha} \Gamma_{\delta \beta}^{\varepsilon}-\Omega_{\delta \gamma{ }^{\top} \Gamma_{\varepsilon \beta}^{\alpha}}^{\alpha} .
$$

On using the expression of $\Gamma_{\gamma \beta}^{\alpha}$ for the various range of indices given in (2.19) and taking account of (2.16) and (2.17), we find that all the particular values of $\left[K^{S}\right]_{\delta \gamma \beta^{\alpha}}$ are

(a) $\left[K^{S}\right]_{k j i}{ }^{k}=K_{k j i}{ }^{k}-C_{k a}{ }^{k} C_{j i}^{a}+C_{j a}{ }^{h} C_{k i}{ }^{a}$

$$
\begin{aligned}
& -\frac{1}{2}\left(C_{k a}{ }^{h} K_{j i b}{ }^{a}-C_{j a}{ }^{h} K_{k i b}{ }^{a}-2 C_{i a}{ }^{k} K_{k j b}{ }^{a}+C_{k 1}{ }^{a} K^{h}{ }_{j b a}-C_{j i}{ }^{a} K^{{ }^{h} b a}\right) y^{a} \\
& +\frac{1}{4}\left(K_{k c d}^{h_{k c d}} K_{j i b}{ }^{d}-K^{h_{i c d}} K_{k i d}{ }^{d}-2 K^{h_{i c d}} K_{k j b}{ }^{d}\right) y^{c} y^{b},
\end{aligned}
$$

(b) $\left[K^{s}\right]_{\bar{k} j i}{ }^{h}=\nabla_{i} C_{k i}^{h}-\nabla^{h} C_{k j i}+\frac{1}{2}\left(\nabla_{i} K^{\left.h_{i a k}\right)} y^{x}\right.$

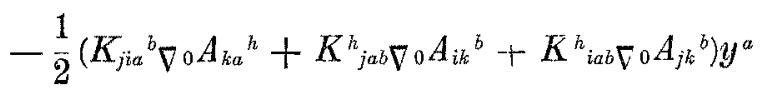

(c) $\left[K^{S}\right]_{k_{j i}^{-j}}{ }^{h}=-\nabla_{i} C_{k j}{ }^{h}+\nabla^{h} C_{k j i}-\frac{1}{2}\left(\nabla_{k} K^{h}{ }_{i a j}\right) y^{a}$

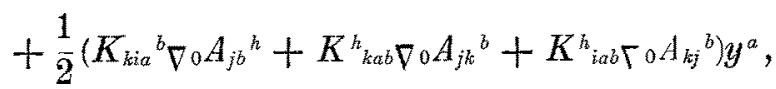

(d) $\left[K^{S}\right]_{k j \bar{i}}{ }^{h}=\nabla_{k} C_{j i}{ }^{h}-\nabla_{j} C_{k i}{ }^{h}-\frac{1}{2}\left(\nabla_{k} K^{h_{j a i}}-\nabla_{j} K^{h}{ }_{k a i}\right) y^{a}$

$$
+K_{k j a}^{b} y^{\alpha} \nabla_{0} A_{b i}{ }^{h} \text {, }
$$

(e) $\left[K^{S}\right]_{\bar{k} \bar{j} i^{h}}=-\frac{1}{2}\left(\partial_{\bar{k}} K^{h_{i a j}}-\partial_{\bar{j}} K^{h_{i a k}}\right) y^{a}-\frac{1}{2}\left(K^{h_{i k j}}-K^{h_{i j k}}\right)$

$$
+C_{k a}{ }^{h} C_{j i}{ }^{a}-C_{j a}{ }^{h} C_{k i}{ }^{a}-\nabla_{0} A_{k a}{ }^{h} \nabla_{0} A_{j a}{ }^{h}+\nabla_{0} A_{j a}{ }^{k} \nabla_{0} A_{k i}{ }^{a}
$$




$$
\begin{aligned}
& -\frac{1}{2}\left(C_{k a}{ }^{h} K^{a}{ }_{i b j}-C_{j a}{ }^{h} K^{a_{i b k}}+C_{j i}{ }^{a} K^{h}{ }_{a b b}-C_{k i}{ }^{a} K^{h}{ }_{a b j}\right) y^{b} \\
& +\frac{1}{4}\left(K^{h}{ }_{a c k} K^{a}{ }_{i b j}-K^{h}{ }_{a c j} K^{a}{ }_{i b k}\right) y^{c} y^{b},
\end{aligned}
$$

(f) $\left[K^{S}\right]_{\bar{k} \bar{j}_{\bar{i}}}{ }^{h}=-\frac{1}{2}\left(\partial_{\bar{k}} K^{h_{j a i}}\right) y^{a}-\frac{1}{2} K^{h}{ }_{j k i} \frac{1}{4} K^{k}{ }_{a c k} K^{a}{ }_{j b i} y^{c} y^{b}$

$$
\begin{aligned}
& +2{ }_{k} C_{j i}{ }^{h}+C_{k a}{ }^{h} C_{j i}{ }^{a}-O_{j a}{ }^{h} C_{k i}{ }^{a} \\
& -\frac{1}{2} C_{k a}{ }^{h} K^{a}{ }_{j b i} y^{b}+\frac{1}{2} C_{k i}{ }^{a} K^{h}{ }_{j b a} y^{b}-\frac{1}{2} C_{j i}{ }^{a} K_{k b a}{ }^{b} y^{b} \\
& +\nabla k \nabla 0 A_{j i}{ }^{h}-\nabla j \nabla 0 A_{k i}{ }^{h}
\end{aligned}
$$

(g) $\left[K^{S}\right]_{k \bar{j}_{\bar{i}}^{-h}}=\frac{1}{2}\left(\partial_{\bar{j}} K^{h}{ }_{k a i}\right) y^{a}+\frac{1}{2} K_{k j i}^{h_{k i}}-\frac{1}{4} K^{h_{a c j}} K^{\alpha}{ }_{k b i} y^{c} y^{b}$

$$
\begin{aligned}
& -\partial_{j} C_{k i}{ }^{h}-C_{j a}{ }^{h} C_{k i}{ }^{a}-C_{k a}{ }^{h} C_{j i}{ }^{a} \\
& +\frac{1}{2}{O_{j a}}^{h} K^{a}{ }_{k b i} y^{b}-\frac{1}{2} C_{j i}{ }^{a} K^{h}{ }_{k b a} y^{b}+\frac{1}{2} C_{k i}{ }^{a} K^{h}{ }_{j b a} y^{b} \\
& -\nabla j \nabla 0 A_{k i}{ }^{b}+\nabla k \nabla 0 A_{j i}{ }^{h},
\end{aligned}
$$

(h) $\left[K^{S}\right]_{\bar{k} \bar{j} \bar{i}^{h}}=\partial_{\bar{k}} \nabla_{0} A_{j i}^{h}-\partial_{\bar{j}} \nabla_{0} A_{k i}^{h}$

$$
\begin{aligned}
& +\left(C_{k a}{ }^{h}-\frac{1}{2} K^{h}{ }_{a b k} y^{b}\right) \nabla_{0} A_{j i}{ }^{a}-\left(C_{j a}{ }^{h} K^{h}{ }_{a b j} y^{b}\right) \nabla_{0} A_{k i}{ }^{a} \\
& -O_{k i}{ }^{a} \nabla_{0} A_{j a}{ }^{h}+C_{j i}{ }^{a} \nabla_{0} A_{k a}{ }^{h},
\end{aligned}
$$

(i) $\left[K^{S}\right]_{k j i}{ }^{\bar{h}}=-\nabla_{k} C_{j i}{ }^{h}+\nabla_{j} C_{k i}{ }^{h}-\frac{1}{2}\left(\nabla_{j} K_{k i b}{ }^{h}-\nabla_{k} K_{j i b}{ }^{h}\right) y^{b}$

$$
-K_{k j b}^{a} y^{b} \nabla 0 A_{a i}{ }^{h},
$$

(j) $\left[K^{S}\right]_{\bar{k} j i}{ }^{\bar{h}}=-\frac{1}{2} \partial_{\bar{k}}\left(K_{j i a}{ }^{h} y^{a}\right)-\partial_{\bar{k}} C_{j i}{ }^{h}-\mathrm{C}_{k a}{ }^{h} C_{j i}{ }^{a}+C_{j a}{ }^{h} C_{k i}{ }^{a}$

$$
\begin{aligned}
& +\nabla j \nabla{ }_{0} A_{k i}{ }^{a}+\frac{1}{2} C_{k i}{ }^{a} K_{j a b}{ }^{h} y^{b}-\frac{1}{2} C_{j i}{ }^{a} K_{k a b} y^{b}-\frac{1}{2} C_{j a}{ }^{h} K^{a}{ }_{i b l} y^{b} \\
& -\frac{1}{4} K_{j a c}{ }^{h} K^{a}{ }_{i b b} y^{c} y^{b}-\nabla{ }_{0} A_{k i}{ }^{a} \nabla_{0} A_{a i}{ }^{h},
\end{aligned}
$$


(k) $\left[K^{S}\right]_{k \bar{j} i^{\bar{b}}}=\mathfrak{d}_{\bar{j}}\left(K_{k i a}{ }^{h} y^{a}\right)+\partial_{j} C_{k i}{ }^{k}+C_{j a}{ }^{h} C_{k i}{ }^{a}-C_{k a}{ }^{h} C_{j i}{ }^{a}$

$$
\begin{aligned}
& -\nabla k \Gamma 0 A_{j i}{ }^{a}-{ }_{2} C_{j i}{ }^{a} K_{k a b}{ }^{h} y^{b}+\frac{1}{2} C_{k i}{ }^{a} K_{j a b} y^{b}+\frac{1}{2} C_{k a}{ }^{b} K^{a b j}{ }^{a} y^{b} \\
& +\frac{1}{4} K_{k a c}{ }^{b} K^{a_{i b j}} y^{c} y^{b}+\nabla_{0} A_{J k^{a}}^{a} \nabla 0 A_{a i}{ }^{h},
\end{aligned}
$$

(l) $\quad\left[K^{S}\right]_{k j \bar{i}} \bar{i}^{\vec{h}}=K_{k j i}{ }^{h}+\frac{1}{4}\left(K_{k c c}{ }^{h} K_{j b i}^{h}-K_{j a c}{ }^{h} K_{k b i}{ }^{a}\right) y^{c} y^{b}$

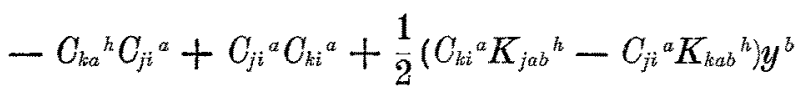

$$
\begin{aligned}
& +\frac{1}{2}\left(C_{k a}{ }^{h} K_{j b i}{ }^{a}-C_{j a}{ }^{h} K_{k b i}{ }^{a}+C_{a i}{ }^{h} K_{k j b}{ }^{a}\right) y^{b},
\end{aligned}
$$

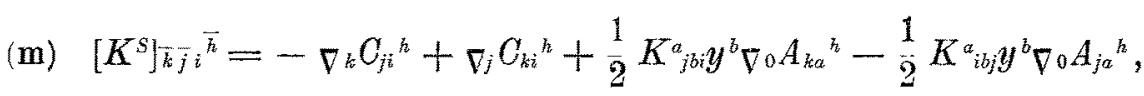

(n) $\left[K^{S}\right]_{\bar{b}} \bar{i}^{\bar{h}}=\nabla_{i} O_{k_{j}}{ }^{h}+\nabla^{h} C_{k j^{3}}-\frac{1}{2} K^{a}{ }_{j b i} y^{b} \nabla 0 A_{k a}{ }^{b}-\frac{1}{2} K_{j a b}{ }^{h} y^{b} \nabla_{0} A_{k \dot{b}}{ }^{a}$,

(o) $\left[K^{S}\right]_{k \bar{j} \bar{i}^{-\bar{h}}}=-\nabla_{i} C_{j k}{ }^{h}+\nabla^{h} C_{j k i}-\frac{1}{2} K^{a_{k} b i} y^{b} \nabla_{0} A_{j a}{ }^{h}-\frac{1}{2} K_{k a b}{ }^{h} y^{b} \nabla_{0} A_{j i}{ }^{a}$,

(p) $\left[K^{S}\right]_{\bar{k} \bar{j} \bar{i}^{\bar{h}}}=\partial_{\bar{k}} C_{j i}{ }^{k}-\partial_{j} C_{k i}{ }^{h}+C_{k a}{ }^{h} C_{j i}{ }^{a}-C_{j a}{ }^{h} C_{k i}{ }^{a}$

$$
+\left(\nabla_{0} A_{k i}{ }^{a}\right) \nabla_{0} A_{j a}{ }^{h}-\left(\nabla_{0} A_{i j}{ }^{a}\right) \nabla_{0} A_{k a}{ }^{h} .
$$

From these it is clear that if the base Finslerian manifold is flat, then $O_{j i}{ }^{h}$ and $K_{k j i}{ }^{h}$. Conseversely being zero, all the components $\left[K^{s}\right]_{\delta \gamma \beta^{x}}$ vanish and hence $T(M)$ is flat. If $\left[K^{S}\right]_{\delta p^{*}}$ vanish, then by taking account of the homo. geneity properties of $C^{\prime} s$ and $K^{\prime} s$, we find, for instance, from $(4.4(j))$ that

$$
\partial_{\bar{k}} C_{j i}{ }^{b}-C_{k a}{ }^{h} C_{j i}{ }^{a}+C_{j a}{ }^{k} C_{k i}{ }^{a}=0,
$$

the left hand side being homogeneous of degree minus two in the $y^{\prime} s$.

Then transvecting (4.5) by $y^{k}$ and by using the relations

$$
y^{k} \partial_{\bar{k}} C_{j i}{ }^{h}=-O_{j i}{ }^{k}, C_{k \alpha}{ }^{k} y^{k}=0,
$$

we obtain

$$
C_{j i}^{h}=0
$$


which implies that the base manifold $M$ is Riemannian, and for the case $\left[K^{S}\right]_{\delta \beta_{y}{ }^{\alpha}}$ given in $(4.4(a) \sim(p))$ reduce respectively to
(a) $\left[K^{s}\right]_{k j i}^{h}=K_{k j i}^{h}+\frac{1}{4}\left(K_{d c k}^{h} K_{j i b}^{d}-K_{d c j}{ }^{h} K_{k i b}^{d}-2 K_{f c i}{ }^{h} K_{k j b}\right) y^{b}$,
(b) $\left[K^{s}\right\rfloor_{k j i}{ }^{h}=\frac{1}{2}\left(\nabla_{j} K_{k a i}{ }^{h}\right) y^{a}$
(c) $\left[K^{S}\right]_{k \vec{j} i^{h}}=-\frac{1}{2}\left(\nabla_{k} K_{j a i}{ }^{h}\right) y^{a}$
(d) $\left[K^{S}\right]_{k j i} \bar{i}^{k}=-\frac{1}{2}\left(\nabla_{k} K_{i a j}^{k}-\nabla_{j} K_{i a k}{ }^{k}\right) y^{a}$,
(e) $\left[K^{S}\right]_{\bar{k}_{\bar{j} i}{ }^{h}}=K_{k j i}{ }^{h}+\frac{1}{4}\left(K_{k c c}{ }^{h} K_{j b i}{ }^{a}-K_{j c a}{ }^{h} K_{k b i}{ }^{a}\right) y^{c} \boldsymbol{y}$,
(f) $\left[K^{S}\right]_{k-\bar{j} \bar{i}^{h}}=\frac{1}{2} K_{k i j}{ }^{h}+\frac{1}{4} K_{k c a}{ }^{h} K_{i b j}{ }^{a} y^{c} y^{b}$,
(g) $\left[K^{S}\right]_{k \bar{j}^{-}{ }^{h}}=+\frac{1}{2} K_{i j k}^{h}-\frac{1}{4} K_{j c \alpha}{ }^{h} K_{k b i}{ }^{a} y^{c} y^{b}$,
(h) $\left[K^{s}\right]_{\vec{k}} \bar{j}_{\bar{i}}^{b}=0$,
(i) $\left[K^{s}\right]_{k j i}{ }^{\bar{b}}=\frac{1}{2}\left(\nabla i K_{k j a}{ }^{h}\right) y^{a}$,
(j) $\left[K^{s}\right]_{\bar{k} j i}^{\bar{h}}=-\frac{1}{2} K_{j i k^{h}}-\frac{1}{4} K_{j a c}{ }^{h} K_{k b i}{ }^{a} y^{c} y^{b}$,
(k) $\left\lfloor K^{S}\right]_{k \overline{j i}} \bar{h}^{\bar{h}}=\frac{1}{2} K_{k j i}{ }^{h}+\frac{1}{4} K_{k a c}^{h} K_{j b i}^{a} y^{c} y^{b}$,

$(4.7)$

(l) $\left[K^{s}\right]_{k_{j} \bar{i}^{-\bar{h}}}=K_{k j i^{h}}{ }^{h}+\frac{1}{4}\left(K_{k a c}{ }^{h} K_{i b j}{ }^{a}-K_{j a c}{ }^{k} K_{i b l}{ }^{a}\right) \boldsymbol{y}^{b}$

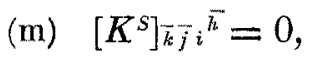

(n) $\left[K^{S}\right]_{\bar{k} j} \bar{i}_{\bar{i}}^{\bar{h}}=0$,

(o) $\left[K^{S}\right]_{k \vec{j}-\vec{i}}-\vec{h}=0$,

(p) $\left[K^{S]_{\bar{k}}-\bar{i} \bar{i}}=0\right.$. 
Thus, if $\left[K^{S}\right]_{\delta \beta \gamma}{ }^{\alpha}$ vanish, then we find from $(4.7(a))$, for example, that $K_{k j f}{ }^{k}$ vanishes because it does not depend upon the $y^{\prime} s$, while the second term in the right hand side of $(4.7(a))$ involves them as a linear combination, and hence the base Riemannian manifold is flat. We have proved

Theorem 12. - If the tangent bundle $T(M)$ over a Finslerian manifold $M$ whose metric is Sasakian lift $g^{s}$ is flat, then $M$ is flat and the converse is true also.

We have from the Equations (4.3) the relation

$$
\left[K^{S}\right]_{\delta \gamma \beta} \beta^{\alpha}+\left[K^{S}\right]_{\gamma \delta \beta^{\alpha}}=0
$$

immediately, into which we substitute $(4.4)$ corresponding to the range of different indices to obtain the sole relation (H. RUND [15], p. 105).

$$
K_{k j i}{ }^{h}+K_{j k i}{ }^{h}=0 .
$$

Also, by taking account of (2.4), we have

$$
\left[K^{S}\right)_{\delta \gamma \beta \alpha}+\left[K^{S}\right]_{\delta \gamma \times \beta}=0,
$$

where we have put

$$
\left[K^{S}\right]_{\delta \gamma \beta \alpha}=g_{\varepsilon \alpha}^{S}\left[K^{S}\right]_{\delta \gamma \beta^{\varepsilon}},
$$

into which we substitute (4.4) to obtain the sole relation (H. RUND, [15], p. 105)

$$
K_{k h j i}+K_{k h i j}+2 C_{j i b} K_{k k a}^{b} y^{a}=0
$$

If in (4.1) we change $X, Y$ and $Z$ cyolically and sum up the results, we have so-called Bianchi's identity, which has in terms of the adapted frame the expression

$$
\begin{aligned}
& {\left[K^{S}\right]_{\delta \beta^{\alpha}}+\left[K^{s}\right]_{\gamma \beta \delta^{\alpha}}+\left[K^{S}\right]_{\beta \delta \gamma}{ }^{\alpha}} \\
& =\delta_{\delta} \Omega_{\gamma \beta}{ }^{\alpha}+\delta_{\gamma} \Omega_{\beta \delta}{ }^{\alpha}+\delta_{\beta} \Omega_{\delta \gamma^{\alpha}} \\
& +\Omega_{\delta \varepsilon}{ }^{\alpha} \Omega_{\gamma \beta}{ }^{\varepsilon}+\Omega_{\gamma \varepsilon}{ }^{\alpha} \Omega_{\beta \delta}{ }^{\varepsilon}+\Omega_{\beta \varepsilon}{ }^{\alpha} \Omega_{\delta \gamma^{\varepsilon}}
\end{aligned}
$$

and by substituting each term of (4.4) in accordance with the various indices that belong to (4.12), we deduce the following four relations (H. RUND, [15], p. 105 and p. 110).

$$
K_{k j i}^{h}+K_{j i k i}^{h}+K_{i k j}^{h}=0
$$




$$
\begin{aligned}
& \nabla_{i} K_{k j l}^{h}+\nabla_{k} K_{j l}^{h}+\nabla_{j} K_{l k i^{h}} \\
& +\left(K_{l k c}^{b} y^{c} \mathfrak{\partial}_{b} \Gamma_{j i}^{a}+K_{k i c}^{b} y^{c} \mathfrak{z}_{b} \Gamma_{l i}^{a}+K_{j l c}^{b} y^{c} \mathfrak{z}_{\bar{b}} \Gamma_{k c}^{a}\right)=0, \\
& \left(\nabla_{l} K_{k j a}{ }^{h}+\nabla_{k} K_{j i a}{ }^{h}+\nabla_{j} K_{l k a}{ }^{h}\right) y^{a} \\
& +\left(K_{l k b} \nabla_{0} A_{j c}{ }^{h}+K_{k j b}{ }^{e} \nabla_{0} A_{l c}{ }^{h}+K_{j l b^{c}}{ }^{c} \Gamma_{0} A_{k c}{ }^{h}\right) y^{b}=0
\end{aligned}
$$

and

$$
\begin{gathered}
\partial_{i} \nabla_{0} A_{j i^{h}}=\Gamma_{k} C_{j i}{ }^{h}+y^{b} \nabla_{b} C_{k j i^{h}} \\
-\left(C_{i a}{ }^{h} \nabla_{b} C_{k j}{ }^{h}+C_{j a}{ }^{h} \nabla b C_{k i}{ }^{a}+C_{j i}{ }^{a} \nabla_{b} C_{k a}{ }^{h}+2 C_{k a}{ }^{h} \nabla_{b} C_{j i}{ }^{a}\right) y^{b} .
\end{gathered}
$$

The formulas (4.13) and (4.14), or equivalently (4.15) are called by H. Rund Bianchi's identitites of the first and second kinds, respectively.

Taking account of the theorem 3 we have from (4.11) and (4.14)

Theorem 13. - If the tangent bundle $T(M)$ over a Finslerian manifold is Kaehlerian, the curvature tensor of the base Finsler manifold satisfies

$$
\begin{gathered}
K_{k h j i}+K_{h h j i}=0 \\
\nabla_{l} K_{k j i}{ }^{h}+\nabla_{k} K_{j l i}{ }^{h}+\nabla_{j} K_{l k i}{ }^{h}=0
\end{gathered}
$$

$\S 5$. - Ricei and scalar curvature of $\nabla^{S}$.

We now compate the Ricci curvature of $r^{s}$. It is given by

$$
\left[K^{s}\right]_{\gamma \beta}=\left[K^{s}\right]_{\delta \curlyvee \beta}^{\delta}
$$

and on using (4.4) those components $\left[K^{S}\right]_{\% \beta}$ will be

(a) $\left[K^{S}\right]_{j i}=K_{j i}-\frac{1}{4}\left(K^{a j c}{ }^{d} K_{a i b d}+2 K^{a_{i c}{ }^{d}} K_{a j b d}+K_{j a c}{ }^{d} K^{a} \cdot{ }_{i b d}\right) y^{c} y^{b}$

$$
\begin{aligned}
& +\frac{1}{2}\left(C_{j}^{a c} K_{c i b a}+C_{i}{ }^{a c} K_{c j b a}-C_{c}{ }^{a c} K_{j i b a}\right) y^{b} \\
& -2\left(C_{b a}^{b} C_{j i}^{a}+C_{j a}^{b} C_{b i}{ }^{a}\right) \\
& -\frac{1}{2} \partial \bar{b}\left(K_{j i a}^{b} y^{b}\right)-\partial_{a}^{-} C_{j i}^{a}+\nabla j \nabla 0 A_{i}-\nabla_{0} A_{b j}{ }^{a} \nabla 0 A_{a i}{ }^{b},
\end{aligned}
$$

(b) $\quad\left[K^{s}\right]_{j i}=-\frac{1}{2} \nabla b\left(K_{j a i}^{b} y^{b}\right)+\frac{1}{2}\left(K_{d j a}^{b} \nabla 0 A_{i b}{ }^{d}+K^{a}{ }_{j b i} y^{b} \nabla_{0} A_{a}\right)$

$$
+\nabla_{i} C_{u j}{ }^{a}-\nabla_{j} C_{a i}{ }^{a}
$$


(c) $\left.\quad\left[K^{S}\right]_{j \bar{i}}=-\frac{1}{2} \nabla_{b}\left(K_{j a i}^{b} y^{b}\right)+\frac{1}{2} K_{d j a}^{b} \nabla_{0} A_{i b}{ }^{d}+K^{a}{ }_{j b i} y^{b} \nabla_{0} A_{a}\right)$

$$
+\nabla_{i} C_{a j}{ }^{a}-\nabla_{j} C_{a i}{ }^{a}
$$

(d)

$$
\begin{aligned}
{\left[K^{s}\right]_{\bar{j} \bar{i}} } & =\frac{1}{4} K^{c a}{ }_{c j} K_{c a b i} y^{c} y^{b} \\
& +\left(\partial_{a} C_{j i}{ }^{a}-2 \partial_{\bar{j}} C_{i a}{ }^{a}+2 C_{a b}{ }^{b} C_{j i}{ }^{a}-2 C_{j a}{ }^{b} C_{b i}{ }^{a}\right) \\
& +\nabla_{a} \nabla_{0} A_{j i}{ }^{a}-\nabla_{0} A_{a \nabla_{0}} A_{j i}{ }^{a} .
\end{aligned}
$$

Now, if $T(M)$ is an Einstein space, that is to say, if

$$
\left[K^{S}\right)_{\beta \alpha}=k g_{\beta \alpha}^{S},
$$

or, by (1.24)

$$
\left[K^{S}\right]_{j i}=k g_{j i},\left[K^{S}\right]_{\bar{j} i}=\left[K^{S}\right]_{j \bar{i}}=0\left[K^{S}\right]_{\bar{j} \bar{i}}=k g_{j i},
$$

then, by taking account of the homogeneity properties of the functions in: volved, we have from the fourth equations of (5.2) and of (5.4)

$$
\begin{gathered}
K^{e a}{ }_{c j} K_{e a b i} y^{c} y^{b}=0, \\
\hat{\partial}_{a} C_{j i}^{a}-2 \partial_{j} C_{i^{a}}^{a}+2 C_{a b}{ }^{b} C_{j i}{ }^{a}-2 C_{j a}^{b} C_{b i}{ }^{a}=0, \\
\nabla a \nabla_{0} A_{j i}^{a}-\nabla_{0} A_{a} \nabla_{0} A_{j i}{ }^{a}=0 .
\end{gathered}
$$

Multiplying (5.6) by $y^{j}$ and taking account of (4.6) we find taht

and consequently

$$
C_{i a}{ }^{a}=0
$$

$$
A_{i}=0
$$

which implies by DEIKE's theorem stated in $\S 2$ that the base manifold $M$ is Riemannian, while we have from (5.5)

$$
K_{j i a}{ }^{h} y^{a}=0
$$

and consequently $M$ is flat. Thus

TheoRem 14. - If the tangent bundle over a Finslerian or Riemannian manifold with Sasakian lift $g^{S}$ as metric is an Einstein space, then the base manifold is fat. 
Let us consider the scalar curvature of $r^{s}$ :

$$
\left[K^{S}\right]=\left[K^{S}\right]_{\alpha}^{\alpha} \text {. }
$$

Then we have from (5.2)

$$
\begin{aligned}
{\left[K^{s}\right]=K } & +2_{\nabla a \nabla_{0}} A^{a}-\nabla_{0} A_{a} \nabla_{0} A^{a}-\nabla_{0} A_{c}{ }^{b a} \nabla_{0} A_{b a c} \\
& -\frac{1}{4} K^{e a}{ }_{c}^{d} K_{e a b d} y^{c} y^{b}-2 \partial_{b} C_{a}^{b a}
\end{aligned}
$$

where

$$
K=K_{j}{ }^{i}, C_{a}^{b a}=g^{c b} C_{a c}{ }^{a}
$$

Thus, if $\left[K^{s}\right]$ vanishes, then by taking account of the homogeneity properties of the functions involved again, we have

$$
K=\Gamma_{0} A_{c}^{b a} \Gamma_{0} A_{b a}{ }^{c}+\nabla_{0} A_{a} \nabla_{0} A^{a}-{ }^{2} \nabla_{a} \nabla_{0} A^{a}
$$

$$
K_{j i a}{ }^{h} y^{a}=0
$$

from which we have

$$
\partial_{a}^{-} C_{b}^{a b}=0 \text {, }
$$

Theorfm 15. - If in the tangent bundle $T(M)$ over a Finslerian manifold $M$ with Sasakian metric $g^{s}$ as metric its scalar curvature varishes, the $T(M)$ is Kaehlerian and if $M$ is a Riemannian manifold, $M$ must be flat.

\section{REFERENCES}

[1] L. BeRwALD, Untersuchung der Krümmung allgemeiner metrischen Räume auf Grund der thren Herschenden Parallelismus, Math. Zeitschr. 25 (1926) 40-73.

[2] C. CARATHEODORY, Variationsrechnung und partielle Differentialgleiehungen erster Ordnung, Berlin und Leibzig (1930)).

[3] E. Cartan, Les espaces de Finsler, Act. Sci. et Ind., Hermann, 193 .

[4] E.T. Davies, Areal spaces, Annali di Mat, 55 (1961) 63-76.

[5] A. Derke, Über die Einsler Räume mit $A_{i}=0$. Arc. Math 4 (1953) 45.51.

[6] J. Douglas, The general geometry of paths. Ann. of Math, 296 (1928) 143.168.

[7] T, C. DOYs, Tensor decomposition with applications to the contact and complex groups, Ann. Math., 42 (1941) 698.722.

[8] L. P. EISENHART, Finsler space derived from Riemann space by contact transformations, Ann. Math., 49 (1918) 227.205. 
[9] L. P. EISENHART and M. S. KNEBELMAN, Invariant theory of homogeneous contact tran. sformations, Ann. Math., 37 (1986) 747-765.

[10] A. KAWAGUCHI, Beziehung zwischen einer metrischen linearen Übertragung und einer nicht-metrischen in einem allgemeinen metrischen hamme, Ned. Akad. Wel. Froe. Ser. 40, (1937) $596-601$.

[11] M.S. Knebeman, Collineations and motuns in the generalized spaces of paths, Amer. Jour. Math, 51 (1929) 527.564

[12] А. Kовауаян and K. Nomiza, Eoundation of differential geometry, 1, Interscience Tract., New York 15 (1969).

[13] Y. Muro, On the connections in the manifold admitting homogeneous contact transfor. mations, Proc. Phys -Math. Soe Japan 20 (1938) 451.457.

[14] T. Oxuво, On the groups of affine collineations in the generalized spaces of paths, I, Tensor N.S., 6 (1956) 14l-158; II, ibb. 7 (1957) 1-17; 1II. ibb. 7 (1957) 18833.

[15] H. Rund, The differential geometry of Finsler spaces, Springer-Verlag, Berlin (1959).

[16] S. SASAxy, On the differential geometry of tament bundles of Riemannian Manifolds, I. Tohoku Math. Jour. 10 (1958) 338.3.4; II, ibb 14 (1962) 146.155.

[17] J. L. SYnge, A generalization of the Riemannian lue-element, Trans. Amer. Math. Soc.: 27 (1925) 61.67.

[18] S. TaCubana and M. Oxumura, on the almost complex structure of tangent bundles of Rienannian spaces, Tohoken Math. Jour 14 (1962) 156-161

119] K. Yano and E.T. Davies, Contact tensor calculus, Ann. Mat. pura ed appl. 37 (1954) 1.36.

[20] K. YANo, The theory of Lie derivatives and its applications, North. Holland. Publ, Amsterdam (1957).

[21] $\mathrm{K}$ YANO and E.T. DAYIES, On the tangent bundles of Finsler and Riemannian manifolds, Rend. Circ. Mat. Palermo, 12 (1963) 221.228.

[22] K. YAno, Differential geometry on complex and almost complex snaces, Periamon Press, New York, (1965).

[23] K Y $\mathrm{ANO}$ and S. KuBayash, prolongations of tensor fields and connections to tangent bundles, I. Jour. Math. Soc. Japan, 18 (1966) 194.210; II, ibd. 18 (1966) 236.246.

[24] K. YANo and T. OKubo, On the tangent bundles of generalized spaces of paths. To appear in Rendiconti di Matematica, Roma. 